\title{
Greenwashing, marketing ecologista y marketing ecológico: el caso de Mattel y Asia Pulp \& Paper
}

Greenwashing, ecologist marketingand green marketing: the case of Mattel and Asia Pulp \& Paper

- María Ruiz Córdoba y José Candón-Mena Universidad de Sevilla, España

$\left(\begin{array}{l}\text { Fecha de recepción: } 17 \text { de noviembre de } 2020 \\ \text { Fecha de aprobación: } 17 \text { de diciembre de } 2020\end{array}\right)$

DOI: http://dx.doi.org/10.15304/ricd.3.13.7265

\section{NOTAS BIOGRÁFICAS}

María Ruiz Córdoba es graduada en Publicidad y Relaciones Públicas por la Universidad de Sevilla, donde realizó su Trabajo de Fin de Grado sobre la teoría y la práctica del greenwashing.

Contacto: mariarcor@gmail.com

José Candón-Mena es profesor del Departamento de Comunicación Audiovisual y Publicidad de la Universidad de Sevilla e investigador del Grupo Interdisciplinario de Estudios en Comunicación, Política y Cambio Social (COMPOLÍTICAS). Doctor en Ciencias de la Comunicación y Sociología y Premio Extraordinario de Doctorado por la Universi dad Complutense de Madrid (UCM).

Contacto:jcandon@us.es

\section{Resumen}

El greenwashing, como estrategia de marketing lucrativo, consiste en el uso de publicidad engañosa y otras técnicas para presentar a las empresas y sus productos como medioambientalmente responsables sin base real y ocultando el verdadero impacto ambiental de su actividad. En este artículo contraponemos el concepto de greenwashing con el de marketing ecológico. Este último, también enmarcado en el marketing lucrativo, implica sin embargo un verdadero esfuerzo para integrar los valores ecológicos en todo el proceso empresarial, realizando cambios reales en la política corporativa para hacer compatibles la reducción del impacto ambiental con los fines de lucro. Definimos también el concepto de marketing ecologista como un tipo de marketing social llevadoa cabo por las ONG ambientalistas y empleado a menudo para desenmascarar las prácticas de greenwashing. Presentamos el caso de estudio de Mattel y Asia Pulp \& Paper, cuyas prácticas de greenwashing fueron denunciadas por ONG como Greenpeace y WWF. Analizamos los diferentes tipos de greenwashing empleados por Mattel y Asia Pulp \& Paper y describimos cómo las ONG señaladas emplearon estrategias de marketing social y ecologista para desenmascarar estas prácticas, forzando finalmente a las compañías a emprender verdaderas acciones de marketing ecológico. Concluimos mostrando la interrelación conflictiva entre el greenwashingy el marketing ecologista. Señalamos como, en el caso analizado, el abandono de la estrategia de greenwashing y la implantación de medidas efectivas de marketing ecológico se realizó de forma reactiva en respuesta a la presión de las ONG. En este sentido apuntamos a que una estrategia proactiva de marketing ecológico resulta más coherente con la filosofía de las Relaciones Públicas, evita daños innecesarios 
a la imagen de las empresas y supone la apuesta más eficaz para garantizar la sostenibilidad a largo plazo tanto de las compañías como del medio ambiente.

\begin{abstract}
Greenwashing, as a lucrative marketing strategy, consists of the use of misleading advertising and other techniques to present companies and their products as environmentally responsible without any real basis and hiding the true environmental impact of their activity. In this article, we compare the concept of greenwashingwith the concept of green marketing. The concept of green marketing outlined in the lucrative marketing, implies however a real effort to integrate ecological values in the whole business process, making real changes in the corporate policy to make the reduction of environmental impact compatible with profit purposes. We also define the concept of green marketing as a type of social marketing carried out by environmental NGOs and often used to unmask greenwashing practices. We present the case study of Mattel and Asia Pulp \& Paper, whose greenwashing practices were denounced by NGOs such as Greenpeace and WWF. We analyse the different types of greenwashing employed by Mattel and Asia Pulp \& Paper, and describe how the aforementioned NGOs employed social and environmental marketing strategies to expose these practices, eventually forcing the companies to take real green marketing actions. We conclude by showing the conflicting interrelationship between greenwashingand ecologist marketing. We point out how, in this case, the abandonment of the greenwashing strategy and the implementation of effective green marketing measures was performed in a reactive manner in response to pressure from NGOs. On this matter, we highlight that a proactive green marketing strategy is more consistent with the philosophy of Public Relations, avoids unnecessary damage to the image of companies and is the most effective way of guaranteeing the long-term sustainability of both, companies and the environment.
\end{abstract}

\title{
Palabras clave
}

Greenwashing, marketing ecológico, marketing social, marketing ecologista, publicidad.

\section{Keywords}

Greenwashing, green marketing, social marketing, ecologist marketing, advertising

\section{Sumario}

1. Introducción

2. Marco teórico

\subsection{Marketing ecológico}

2.2. Marketing ecologista

\subsection{Greenwashing}

\section{Metodología}

4. Estudio de caso

4.1. Mattel y Asia Pulp \& Paper vs Greenpeace y WWF

4.2. Modalidades de greenwashingempleadas por Mattel y Asia Pulp \& Paper

5. Conclusiones 
Summary

1. Introduction

2. Theoretical framework

2.1. Green marketing

2.2. Ecologist marketing

2.3. Greenwashing

3. Methodology

4. Case study

4.1. Mattel and Asia Pulp \& Paper vs Greenpeace and WWF

4.2. Greenwashing modalities used by Mattel and Asia Pulp \& Paper

5. Conclusions 


\section{INTRODUCCIÓN}

La práctica del greenwashing como estrategia de marketing basada, entre otras tácticas, en el uso de publicidad engañosa que pretende presentar a las empresas y sus productos como medioambientalmente responsables sin base real y ocultar el verdadero impacto ambiental de su actividad, está cada vez más extendida debido a la creciente conciencia medioambiental de los consumidores (Calomarde, 1995) y de la sociedad en general (Castells 2009, p. 398-443). En este artículo se define el greewashing y sus distintas tipologías como una estrategia del marketing comercial a partir de otros conceptos como el marketing ecológico o el marketing ecologista. El marketing ecológico y el greenwashing se enmarcan en el ámbito empresarial como tipos de marketing comercial con fines lucrativos. Se diferencian en que el marketing ecológico conlleva un verdadero esfuerzo por parte de la empresa para integrar los valores ecológicos y hacerlos compatibles con los legítimos fines de lucro, lo que implica una verdadera transformación en la política empresarial que afecta a todos los elementos del marketing-mix (producto, precio, distribución y promoción). Por contra, el greenwashing solo busca el lucro y no implica ninguna práctica medioambiental real. El marketing ecologista se encuadraría como un tipo de marketing social y no lucrativo llevado a cabo en el ámbito de las organizaciones del tercer sector social y supone la incorporación de los conceptos y estrategias del marketing al ámbito de las organizaciones del tercer sector con el objetivo de alcanzar sus fines sociales, en este casola defensa del medio ambiente.

Partimos de la hipótesis de que, aunque el marketing ecológico sea el modelo de negocio a seguir si el objetivo es la sostenibilidad largo plazo -no solo medioambiental sino también económica-, la realidad es que numerosas empresas llevan a cabo prácticas de greenwashing anteponiendo la rentabilidad a corto plazo y mostrando una actitud irresponsable ante el medio ambiente y la sociedad. Por otra parte, las organizaciones sociales y ONG ambientalistas utilizan el marketing ecologista para denunciar estas prácticas $y$, en particular, desmontar las estrategias engañosas de greenwashing.

Nuestro objetivo es enmarcar dichos usos del marketing en los conceptos señalados de marketing ecológico, ecologista y greenwashing, así como ilustrar las distintas tipologías en cada una de dichas categorías con ejem plos concretos. Se pretende contrastary comprobar si hay corres- pondencia entre lo que las empresas hacen y lo que dicen que hacen, señalando además el importante papel de las organizaciones ecologistas para desvelar las prácticas de greenwashing.

Concluimos señalando cómo numerosas empresas continúan llevando a cabo prácticas de greenwashing con el objetivo de proyectar una imagen verde y comprometida con el medio ambiente u ocultar el impacto ecológico de su actividad, a menudo como respuesta a las denuncias públicas de organizaciones sociales y ambientalistas que hacen uso de estrategias de marketing ecologista. Argumentamos que, a largo plazo, las tácticas de greenwashing resultan insostenibles y contraproducentes en el contexto de una ciudadanía cada vez más concienciada respecto a la sostenibilidad ambiental y ante el empuje de organizaciones sociales y ecologistas que, en muchas ocasiones, logran desmantelar el discurso verde y engañoso del greenwashing, con el consiguiente coste en términos de imagen y reputación para las empresas señaladas. No obstante, la laxa legislación yel poder económico y mediático de grandes empresas hace que, en otras tantas ocasiones, la búsqueda a toda costa de beneficios lucrativos les lleve a preferir asumir el riesgo de las denuncias o tratar de contrarrestarlas, mediante campañas publicitarias y de relaciones públicas, en lugar de asumir los costes de una verdadera política medioambientalmente responsable.

\section{MARCOTEÓRICO}

El marco teórico se centra en tres conceptos básicos: marketing ecológico, marketing ecologista y greenwashing, con objeto de mostrar las diferencias existentes entre ellos.

\subsection{MARKETING ECOLÓGICO}

El marketing ecológico (Coddington, 1993; Peattie, 1995; Calomarde, 2000; Chamorro, 2001), también llamado marketing verde o ambiental, es un enfoque en el que se atiende al respeto al medio ambiente de forma transversal a toda la estrategia empresarial. De esta forma, la empresa adecua su actividad para que esta sea lo más sostenible posible, lo que supone esfuerzos reales y modificaciones respecto: Al diceño del producto o servicio; Una política de precios que, además de garantizar la rentabilidad, tenga en cuenta todas las posibles externalidades ne- 
gativas de su actividad; El uso de canales de distribución sostenibles, por ejemplo acortando dichos canales para promover el comercio de proximidad o usando transportes no contaminantes; $Y$ una promoción responsable que, pudiendo comunicar las medidas medioambientales y promocionar la sostenibilidad del producto para aumentar su valor añadido, no exagere los esfuerzos y los logros en este sentido y sea veraz respecto al impacto ambiental de la actividad de la empresa en cuestión. Se incluirían en este enfoque no solo a las empresas que comercializan productos ecológicos, sino también a aquellas que venden productos o desarrollan actividades con cierto impacto ambiental pero que se esfuerzan por reducir al mínimo dicho impacto, incorporando realmente la ecología a la estrategia empresarial y de marketing.

Según Santesmases (2012) el marketing puede ser entendido como filosofía o como técnica. Como filosofía es una forma de concebir la función comercial o relación de intercambio por parte de la empresa o entidad que ofrece sus productos o servicios al mercado. Esta concepción parte del conocimiento de las necesidadesy deseos del consumidor con el fin de satisfacerlos del modo más beneficioso tanto para el consumidor como para la empresa y la sociedad. Como técnica, el marketing es el modo específico de ejecutar o llevar a cabo la relación de intercambio, que consiste en identificar, crear, desarrollar y servira la demanda.

Por su parte, siguiendo a Calomarde (2000), el marketing ecológico no es más que el modo de planificar y ejecutar la relación de intercambio con la intención de satisfacer tanto a la sociedad en general como al entorno natural. Este tipo de marketing tiene, además, el propósito de responder a las demandas de los clientes de un modo ambientalmente responsable sin dejar de lado la rentabilidad financiera.

La presión de la competencia o las nuevas necesidades de los consumidores hacen necesario el rediseño, sustitución o eliminación de los productos actuales (Calomarde, 2000), lo que requiere una completa reorientación del marketing mix que afecta al producto, el precio, la distribución y la promoción (McCarthy \& Perreault, 1984).

En cuanto al producto, una empresa orientada al marketing ecológicotiene la intención de disminuir los daños ambientales durante la totalidad del ciclo de vida de sus productos. Su objetivo es que la suma de los impactos generados durante la fase de extracción de la materia prima, de producción, de distribución, de uso o consumo y de reutilización, reciclaje o eliminación de residuos sea menor que la del resto de productos que satisfacen la misma necesidad (Chamorro, 2001, p. 6). Para ello, Wicke (1990) propone utilizar materias primas que no escaseen, materiales poco o menos contaminantes, reutilizar envases y que sean del menor volumen posible, retrasar la obsolescencia estilística, funcional y material aumentando así la durabilidad, facilitar la recogida de desechos peligrosos y facilitar la incineración o depósito, entre otros (Muñoz, 2013).

Respecto al precio, el marketing ecológico tiene en cuenta tres factores principales: los costes unitarios, la percepción de los consumidores y la competencia. De acuerdo con Muñoz (2013), respecto a los costes el objetivo de la fijación de precios no es otro que la rentabilidad, ya que para promover un consumo más sostenible es necesario conseguir rentabilidad a largo plazo de las empresas que promueven dichos productos. En cuanto a la competencia, Muñoz propugna la viabilidad de una estrategia que marque precios superiores con respecto a esta, dado que al ser productos más ecológicos los costes son mayores y se necesitan precios superiores para mantener el margen de beneficios. Esta estrategia, además, está ligada al último factor, ya que un precio elevado se relaciona en la mente del consumidor directamente con la calidad, por lo que se utiliza la ecología como valor añadido. Así, la calidad y beneficios que el consumidor percibe del producto pueden motivar la compra de productos más ecológicos a pesar de su precio, pues este está fijado en consonancia con todo el esfuerzo realizado en el ciclo de vida del producto y el consumidor debe ser consciente de ello.

En lo que se refiere a la distribución, según Calomarde (2000), el canal de distribución en el marketing ecológico incluye como objetivos el tratamiento de los residuos de envase y embalaje, incorporando canales inversos efectivos. Así, mientras que en el modelo de negocio tradicional el flujo de los productos sigue la dirección fabricante-distribuidor-cliente, el marketing ecológico tiene en cuenta la dirección inversa, esto es, el retorno de los envases para su reutilización, apostando por un flujo bidireccional a medida que los costes de eliminación sean menos soportables para el consumidor. Los consumidores serán cada vez más proclives a comprar a Fabricantes o distribuidores que acepten el antiguo producto como parte del trato (Calomarde, 2000, p. 22). Para esta recogida de envases y residuos se utilizan los Sistemas Integrados de Gestión (SIG) que simplifican la logística inversa. En cuando al transporte y su impacto evidente en la 
distribución, el consumo de productos de proximidad o el uso de medios de transporte no contaminantes son algunas de las principales estrategias de distribución del marketing ecológico.

Finalmente, respecto a la promoción, Muñoz (2013) establece que la comunicación ecológica que realiza la empresa por medio de la publicidad, las relaciones públicas, la venta personal, promoción, marketing directo, patrocinio y mecenazgo, etc., debe incidir en mantener informado al cliente sobre aspectos relacionados con el medio ambiente, de manera que la empresa y el producto ecológico resulten creíbles. La comunicación de la empresa incorpora así el objetivo de transmitir ideas que apoyen cambios positivos en materia ambiental: reducir el consumo de enerǵa y usar fuentes renovables, reciclar, mejoras medioambientales, programas comunitarios de ecología, valores ecológicos internos dentro de la organización, etc. Para ello, Calomarde (2000) incide en que los mensajes en una organización pro-ecológica deben ser claros, poseer verificación independiente, por ejemplo, a través del etiquetado ecológico, y comunicar los valores de la organización. También advierte de que muchas empresas emplean etiquetas buscando la manera de parecerse lo máximo posiblea las etiquetas oficiales con la intención de confundir al consumidor. De cualquier forma, es importante que el consumidor esté informado, perciba las consecuencias ecológicas de sus hábitos de compra y esté dispuesto a cambiar de hábitos para contribuir a las soluciones medioambientales (Calomarde, 2000).

\subsection{MARKETING ECOLOGISTA}

Mientras que el marketing ecológico parte desde una perspectiva empresarial, denominamos marketing ecologista a aquel que parte desde una perspectiva social, la de las organizaciones madioambientalistas que promueven el ecologismo e incorporan las estrategias de marketing para dichos fines. Se trata por tanto de un tipo de marketing social (Kotler y Roberto, 1991; Andreasen, 1994; Pérez, 2004; Leal, 2000 y 2004) que en este caso pretende modificar conductas perjudiciales para el medioambiente.

Kotlery Zaltman (1971) definen el marketing social como el diseño, ejecución y control de los programas que buscan incrementar la aceptación de una idea social y que conllevan consideraciones sobre producto, precio, distribución, comunicación e investigación de mercado. Consiste por tanto en la aplicación del marketing genérico a un tipo específico de problemas donde el objeto del agente de marketing es cambiar el com portamiento social para beneficiar a la audiencia ya la sociedad en general (Andreasen, 1996). Esta aplicación del marketing a las causas sociales tiene defensores (Kotlery Levy, 1969) y detractores (Luck, 1974; Bartels, 1974), pero en cualquier caso es una realidad en las principales organizaciones del tercer sector. Así, la definición del marketing social conlleva la condición necesaria de la existencia de una causa social (Montero, 2003) y se concibe como una estrategia para cambiar la conducta (Kotler y Roberto, 1991) poniendo por tanto el énfasis en el cambio de comportamiento.

Para Kotler y Roberto (1991) el marketing social de una organización conduce todos sus esfuerzos hacia un grupo (agente de cambio), el cual intenta persuadir a otros (adaptadores o mercado meta) a que acepten, modifiquen o abandonen ciertas ideas, actitudes, prácticas y comportamientos con fines sociales. Se enfatiza el concepto básico de intercambio, un concepto mucho más amplio que el de las meras transacciones de mercado. De esta forma el factor que se intercambia no son solo productos comerciales sino también ideas, creencias, actitudes o pautas de comportamiento de las personas. Por su parte, Pechmann (2002) introduce nuevos conceptos al marketing social, definiéndolo como el proceso de promoción para cambiar el comportamiento individual con el fin de aliviar los problemas sociales, un proceso que incluina las cuatro $P$ del marketing mix (Producto, Precio, Distribución o placement y Promoción), así como la investigación de la audiencia, su segmentación, el análisis competitivo y el enfoque en el intercambio (Pérez, 2004, p. 5).

\subsection{GREENWASHING}

Mientras que el marketing ecológico es una buena práctica de las empresas que apuestan por políticas sostenibles, incluyendo modificaciones y objetivos ecológicos en el producto, precio, distribución y promoción, y el marketing ecologista es un tipo de marketing social propio de las organizacionesecologistas, que incorporan las estrategias del marketing para promover causas sociales y en este caso medioambientales, el greenwashing (Siano, Vollero, Conte \& Amabile, 2017) no es más que una estrategia del marketing comercial con fines lucrativos. Esta consiste en promocionar a la empresa y sus productos como social y medioambientalmente 
responsables, pero sin llevar a cabo esfuerzos reales en favor de la sostenibilidad o exagerando dichos esfuerzos en su comunicación comercial con el fin de lavar la imagen de las compañías, minusvalorar $u$ ocultar su verdadero impacto ambiental o directamente tratar de mejorar su notoriedad e imagen o el posicionamiento de sus productos asociándolos a atributos ecológicos que les otorgan valor añadido pero que no se corresponden con una verdadera política de sostenibilidad en la empresa.

Según García (1999), la posición de las empresas en lo que respecta al medio ambiente se resume en la afirmación de que es más fácil pagar una multa. De la investigación llevada a cabo por la Fundación ETNOR que García (1999) menciona, se sustrajo que las malas prácticas empresariales que dañaban al medioambiente eran habituales y que muchas empresas eran conscientes de ello.

Según Alejos (2013), el término greenwashing proviene de whitewashing que en español seńa blanqueo de dinero, junto con la connotación green o verde en español. Fue el reportero ambientalista americano Jay Westerveld quien acuñó el término en los 80 . Tras observar la intención de los hoteles de ahorrar agua con el objetivo aparente de ayudar a la naturaleza, descubrió que en realidad perseguían la reutilización de las toallas, ya que supuestamente no las lavaban a diario, denunciando el engaño y los fines y puramente comerciales de las empresas hosteleras.

El diccionario inglés de Oxford define el greenwashing como "desinformación diseminada por una organización para presentar una imagen pública respetuosa del medioambiente". Esta definición se podría complementar con la desarrollada por Greenpeace (2008), que abarca no solo la imagen pública de la empresa sino también los bienes que produce, siendo el greenwashing "el acto de engaño al consumidor para que la percepción de los productos y los objetivos de una empresa sean vistos como ecológicamente amigables" (citado en Alejos, 2013, p. 8).

Sobre esta disonancia entre lo que hacen las empresas y lo que dicen que hacen en su comunicación comercial y corporativa, García (1999) argumenta que el coste económico, la inmoralidad de las empresas y el gobierno son los principales responsables de dicha disonancia. De esta forma muchas empresas preferirían incluir la multa a los costes del producto antes que incorporar el coste de una política verdaderamente sostenible, dado el elevado precio de este proceso. Abundaría también la justificación de estas prácticas alegando una pérdida de ventaja competitiva respecto a la actuación desleal de otras empresas que tampoco llevan a cabo una política medioambientalmente responsable.

Según Pistilli (2015), las actividades del greenwashing pueden dividirse en seis tipos. En primer lugar, la prueba faltante, en la cual la organización genera confusión ocultando o no siendo clara en la información que difunde del producto supuestamente ecológico. En segundo lugar, el concepto ambiguo, es decir cuando la organización no es clara respecto a los atributos de los productos supuestamente ecológicos que promociona, optando por imágenes y frases ambiguas. En tercer lugar, la autoglorificación, mediante la cual la empresa se vanagloria en su comunicación de una política ambientalista irreal o exagera sus esfuerzos y logros en este sentido. En cuarto lugar, la falsa certificación, por medio de la cual las empresas se atribuyen a ellas mismas méritos ambientalistas cuando en realidad carecen de certificación autorizada. En quinto lugar, los datos irrelevantes, de forma que la comunicación se centra en mayor medida en productoso atributos intrascendentes en lugar de comunicar los más importantes. Y, finalmente, el menor de los males, estrategia por la cual las empresas se conforman con proclamarse como más ecológicas que la competencia, sin siquiera demostrar este hecho.

Por su parte, Seele y Gatti (2015) identifican cuatro categorías de greenwashing. Greenwashing falso, cuando una empresa acusa falsamente a otra por llevar a cabo malas prácticas referidas al medioambiente, lo que puede tener consecuencias devastadoras para la imagen de la competencia. Greenwashing genuino, que por el contrario hace referencia a la acusación que una organización impone hacia otra, mediante pruebas evidentes. El greenwashing potencia sería la práctica exitosa de la estrategia de greenwashing por la cual una empresa logra engañar al consumidor en lo que respecta a sus políticas ambientalistas o los atributos ecológicos de sus productos, a pesar de no corresponderse con la realidad, beneficiándose del valor añadido que aporta la ecología pero sin asumir los costes de una verdadera política maedioambiental. Por último, el greenwashing inexistente se daría cuando la organización se preocupa verdaderamente por el medioambiente e introduce estos principios ecológicos en su responsabilidad social corporativa, lo que ya hemos definido como marketing 
ecológico y por lo tanto no entraría en la definición de greenwashing.

Por otro lado, la ONG ambientalista Greenpeace en su campaña "Stop greenwashing" (Greenpeace, 2008, citado en Alejos, 2013, p. 8) clasifica las prácticas de greenwashing en cuatro tipologías. Negocio sucio (dirtybusiness), queconsiste en promover un producto o programa como ambientalmente amigable cuando el núcleo de la actividad empresarial es mayormente insostenible y contaminante. Publicidad engañosa (ad bluster) que incluiría a la publicidad y campañas focalizadasen exagerar un logro ambiental con el fin de distraer la atención de problemas ambientales, así como los casos en los que el coste de dichas campañas publicitarias excede sustancialmente los costes de realizar conductas realmente sostenibles. Giro político (political spin), consistente en la práctica de enunciar compromisos y declarar intenciones verdes por parte de la empresa mientras que, paralelamente, se ejercen acciones de lobby para influir en contra de regulaciones medioambientales. Y obedecer la ley, lo que radica en la acción de señalar como un logro voluntario conductas que en realidad son exigidas legalmente.

En definitiva, podemos definir el marketing ecológico y el greenwashing como dos estrategias empresariales de marketing lucrativo, con la importante diferencia de que la primera comprende un esfuerzo real en materia medioambientaly difunde una comunicación veraz, conjugando los legítimos intereses lucrativos con la responsabilidad empresarial en materia de medio ambiente, mientras que la segunda no es más que una estrategia de propaganda éticamente reprochable y que lleva a cabo una comunicación engañosa, primando solo el interés lucrativo incluso por encima de criterios éticos. El marketing ecologista, sin embargo, se enmarcaría como un tipo de marketing social sin fines lucrativos llevado a cabo en el ámbito de lasorganizaciones deltercer sector. No obstante, podríamos mencionar la posibilidad de una convivencia del marketing ecológico y ecologista, por ejemplo en empresas del tercer sector económico o la economía social que comercializan productos ecológicos (marketing ecológico) pero que, además, tanto por su carácter no lucrativo como por su posible vinculación con organizaciones del tercer sector social tienen como misión la defensa del medioambiente, para lo que también utilizan las herramientas del marketing (marketing ecologista), siendo la actividad empresarial un instrumento al servicio de sus fines sociales (por ejemplo, la cooperativa SomEnergía).

\section{METOdOLOGÍA}

Metodológicamente, utilizaremos un estudio de caso (case study) (Yin, 2003), que nos pemite explorar fenómenos destacables por su carácter paradigmático, a la vez que estudiar su contexto (Martínez, 2006). El caso estudiado no constituye una muestra representativa del universo analizado, sino más bien orientativo de ciertas tendencias (Flyvbjerg, 2006) y nos sirve para ilustrar los distintos conceptos utilizados, exponiendo ejemplos de forma descriptiva. Los datos del caso se han extraído mediante la revisióny análisis documental (MacDonald \& Tipton, 1993; Hart, 2008). Dadas las peculiaridades del objeto de estudio, que se mueve entre la teoría y la práctica, el análisis documental se basa en páginas web, noticias en prensa, contenidos publicitarios, informes profesionales, textos legales, etc., tanto de las empresas analizadas como de otros actores implicados, como las ONG ambientalistas, lo que se complementa con una revisión de bibliografía académica de referencia para la construcción del marco teórico. De esta forma se describirá y analizará el caso de las empresas Mattel y Asia Pulp \& Paper tratando de enmarcar sus prácticas en las distintas tipologías de greenwashing.

\section{ESTUDIO DE CASO}

\subsection{MATTEL Y ASIA PULP \& PAPER VS GREENPEACEY WWF}

La Asociación Española de Fabricantes de Juguetes (s.f.) recoge en su página web la siguiente información:

\begin{abstract}
Mattel es una compañía estadounidense que nació en 1945 en California con el propósito de reinventar el mundo del juguete y ofrecer a los niños nuevas maneras de jugar y sobre todo de divertirse. [...] Esta empresa llega a España en 1983, alcanzando en la actualidad el liderazgo en el sector teniendo siempre presente sus valores: "Play Fair", Play Together", "Play with Passion", "Play to Grow". O lo que es lo mismo: "Jugar Limpio", "Jugar Juntos", "Jugar con Pasión", "Jugar para Crecer".
\end{abstract}

Además, como recoge la propia web de Mattel (s.f.), esta empresa se presenta como una compañía muy comprometida con la sociedad. Un ejemplo de ello es su compromiso con la discapacidad intelectual, con iniciativas como las Olim- 
piadas Especiales de 2005. La estrategia comunicativa de Mattel gira en torno a la diversidad e inclusión. Su idea clave es que todo el mundo juega, independientemente de la etnia, raza, religión o género, por lo que presume de apostar por la diversidad (Mattel, s.f.). Respecto a la sostenibilidad, Mattel fabrica el primer producto de plástico reciclado de la marca, hecho de plásticos a base de caña de azúcar, el paquete Rock-aStack de Fisher-Price. Según Ynon Kreiz, presidente y CEO de Mattel:

La sostenibilidad ambiental es una prioridad corporativa en Mattel y la creación de productos y embalajes sostenibles es una parte importante de nuestro compromiso con el planeta (Mattel, 2019).

Mattel necesita una empresa que le abastezca de papel para empaquetar sus productos y este proveedor es Asia Pulp \& Paper (AP\&P), empresa destinada a la fabricación de pulpa y papel que abastece también a otras compañías jugué teras como Disney, Hasbro o LEGO. Sin embargo, Mattel y Asia Pulp \& Paper se vieron inmersos en polémicas por el impacto ambiental de la papelera en el año 2011 tras las denuncias de Greenpeace, WWF o la Asociación Geoinnova, entre otras ONG.

Como expone Greenpeace (2011), la base del problema radica en la procedencia de las cajas de papel que utilizaba la marca Mattel para envolver o empaquetar a la Barbie y otros juguetes. Greenpeace analizó las fibras de celulosa de cajas de grandes marcas de juguetes dando como resultado una mezcla de maderas tropicales procedentes de Indonesia. Es decir, la actividad empresarial de Asia Pulp \& Paper destruía selvas vírgenes de Indonesia para extraer el papel que Mattel utilizaba para empaquetar sus juguétes, atentando contra la flora y fauna local, incluyendo sus bosques tropicales y animales en extinción como el tigre de Sumatra, orangutanes y elefantes.

\section{Imagen 1. Deforestación en Indonesia}

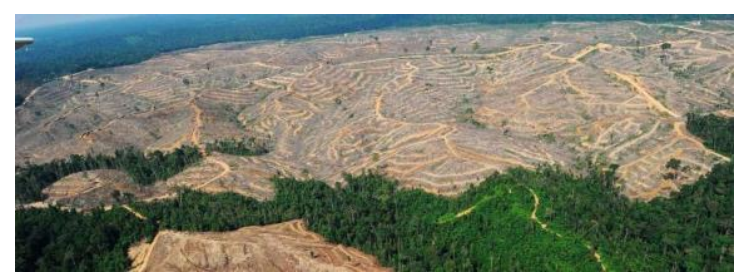

Fuente: https://bluavoluntariado.org/blog/voluntariadoen/indonesia/la-selva-de-indonesia-en-peligro/.

El 7 de junio de 2011 Greenpeace lanzó una campaña dirigida a más de 40 países centrada en la icónica imagen de Barbie, la marca estrella de Mattel. El eje creativo de la campaña de Greenpeace gira en torno a la ruptura de Ken y Barbie, tras descubrir este que Barbie está implicada en la destrucción de bosques de Indonesia (Greenpeace, 2011a y 2011b).

\section{Imagen 2. Campaña de Greenpeace}

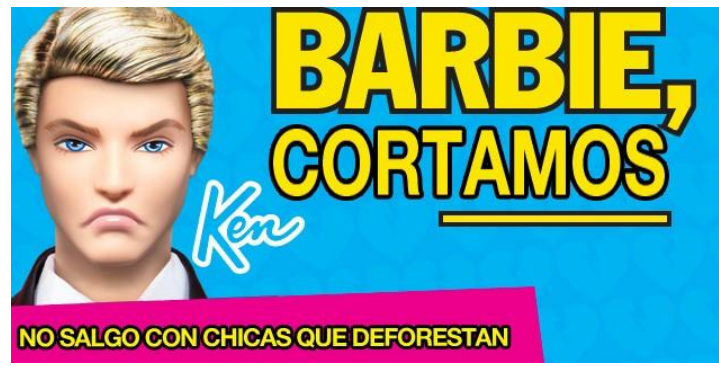

Fuente: https://cutt.ly/cy1Krgq

La respuesta del públicoa dicha campañafue todo un éxito, obteniendo una gran repercusión según la propia ONG (Soto, 2011a). Tras el lanzamiento, Greenpeace organizótambién en España acciones de street marketing (Greenpeace, 2011c). Activistas de Greenpeace se lanzaron a varias tiendas de juguetes de Madrid y colocaron etiquetas en los juguetes de Mattel (González, 2011). Greenpeace también recoge firmas a una petición para proteger los bosques de Indonesia (Greenpeace, s.f.).

La Asociación Geoinnova, por su parte, creó la campaña Forest Heroes (Geoinnova, s.f.). Con ella se pretende proteger los bosques del mundo mediante la integración de activistas, comunidades locales y ciudadanos individuales que desarrollan actividades para proteger estos bosques. Su último proyecto fue la realización de un vídeo utilizando un dron para mostrar la deforestación que sufren los bosques de Indonesia.

También WWF mantiene activa la lucha por proteger los bosques de Sumatra y la extinción del orangután borneano y el orangután de Sumatra, consecuencia de la deforestación en Indonesia (WWF, s.f. a). La ONG lleva desde el año 2000 monitoreando junto con otras entidades los impactos negativos de la deforestación y en 2004, en alianza con ONG locales de la provincia de Riau, estableció Eyes on the Forest, una coalición de ONG ambientales para investigar la tala de bosques (WWFet. al., s.f.).

Mattel reaccionóa estas denuncias anunciando una nueva política de "adquisición sostenible" para todos sus productos que aborde el asunto de la deforestación, además de nuevos requisitos 
para sus proveedores de envases que incluyen pautas medioambientales (Mattel, 2011). Asia Pulp \& Paper, por su parte, argumentó en un primer momento que la denuncia de Greenpeace carecía de sentido, acusando al grupo ecologista de ser "irresponsable por jugar con los sentimientos de los niños y sus padres y de repetir las mismas alegaciones infundadas de siempre, atacando en el proceso a la industria de una nación en vías de desarrollo" (Europa Press, 2011; Comunicarse, 2011).

El 5 de octubre de 2011 Greenpeace lanza un comunicado de prensa (Greenpeace, 2011d) celebrando la decisión de Mattel ante sus cambios en la política medioambiental, en la que excluye los productos papeleros fabricados por empresas vinculadas con la deforestación. Usando su habitual estrategia del dominó (de Lestrade, 2012), Greenpeace centró sus denuncias en una de las grandes em presas como Mattel con el fin de que una vez lograra forzarla a realizar cambios le siguieran el resto de empresas, como Disney, Hasbro y LEGO, dado que en general prefieren evitar ser el siguiente blanco de las campañas de la ONG y el consecuente deterioro de su imagen corporativa.

Como consecuencia, en España, empresas como Unipapel, Inapa Ibérica, Ebix o Comart rompieron su vínculo comercial con $A P \& P$, rechazada también por la mayor parte del sector editorial, desde Susaeta o Salvat hasta Random House Mondadori. Ante tal desgaste de imagen y comercial, Asia Pulp \& Paper invierte grandes cantidaddes de dinero en lavado de imagen, por ejemplo, lanzando acciones solidarias dedicadas a proteger el rinoceronte, construir una escuela, regalar El Corán en vísperas del Ramadán, etc. Además, en 2013 anunció su nueva "Política de Conservación de Bosques" y en 2015 estableció su "Política de Manejo Forestal Sostenible 2.0". Ante estos avances Greenpeace decidió parar su campaña contra AP\&P (Maitar, 2013). Sin embargo, perdura en España su mala fama porsu falta de compromiso medioambiental, aun teniendo el sello PEFC (Soto, 2011b). Así, AP\&P se vio Inmersa en numerosos incendiosen las turberas de las islas de Sumatra y Borneo (WWF, s.f. b), produciéndose unos 130.000 focos de incendios. Investigaciones de Greenpeace aseguran que las empresas de aceite de palma y de papel trabajaron rápido para sacar provecho de la devastación mediante el establecimiento de plantaciones en áreas quemadas. El 5 de febrero de 2018, con motivo del $\mathrm{V}$ aniversario de la Política de Conservación Forestal de Asia Pulp \& Paper, varias ONG como AURIGA, Forest Peoples Programme, Haki, Jikalahari, Warsi, Rainforest Action Network,
Titian, Walhí Riau, Wetlands International y WWF denunciaron conjuntamente que el compromiso de la empresa no ha avanzado lo suficiente (WWF et al., 2018).

Finalmente, en el informe "APP acknowledges links to controversial suppliers, but fails to release an auditor's report" de la Anti Forest Mafia Coalition, AP\&P confirma la veracidad de las acusaciones por parte de varias ONG así como la destrucción de turberas e incendios incluso después de haberse comprometido en 2013 a cero deforestaciones (Koalisi Anti Mafia Hutan, 2019).

El cambio de estrategia y posicionamiento sostenible de AP\&P, reconociendo su rersponsabilidad tras el informe, muestra que no les ha quedado más remedio que reposicionar su marca y su empresa. Solo un año más tarde del escándalo de Indonesia de 2011, AP\&P inicia la hoja de ruta de sostenibilidad de la empresa, con la clara intención de fortalecer una imagen más sostenible tras el escándalo, aunque aún le quedan muchos asuntos pendientes y en estos años ha combinado avances verdaderos con campañas de greenwashing que exageran sus logros o invisibilizan los retos pendientes.

\subsection{MODALIDADES DE GREENWASHING EMPLEADAS POR MATTEL Y ASIA PULP \& PAPER}

Siguiendo a Pistilli (2015), Seele y Gatti (2015) y Greenpeace (2008), podemos encuadrar las prácticas de AP\&P en distintas modalidades de greenwashing.

Atendiendo a la clasificación de Pistilli (2015, citado en Salas, 2018), Asia Pulp \& Paper incurriría en la estrategia de "prueba faltante", ya que genera confusión ocultando o no siendo clara en la información que difunde. Por ejem plo, AP\&Pse comprometió en 2014 a restaurar un millón de hectáreas sin contar con un plan firme para esta restauración. También ocultó vínculos corporativos con 27 proveedores e incluso engañó a sus accionistas ocultando la verdadera producción de su fábrica de $\mathrm{OKI}$ ante la inexistencia de pruebas firmes del suministro de madera.

En segundo lugar, $A P \& P$ hace uso del "concepto ambiguo", ya que no es clara con los atributos de sus productos, optando porimágenes y frases ambiguas. Por ejemplo, AP\&Pafirmó en su momento su compromiso de mejora y atención con las comunidades indígenas sin concreción de 
medidas e incumpliendo promesas a las comunidades de los territorios afectados.

Finalmente, AP\&P incurre en la "autoglorificación" ya que se galardona ella misma como ambientalista, por ejemplo, proclamando con orgullo su "Política de Conservación de Bosques" en 2013 o el retiro de 7000 hectáreas en 2015 . Sin embargo, ese mismo año se produce una sucesión de incendios en Indonesia en la que AP\&P podría estar involucrada. También, denotando su falta de credibilidad, construye una nueva fábrica de celulosa en Indonesia en 2016 y otra en la India en 2019, con las que aumentaría el abastecimiento de manera virgen, o incumple su PCF al no cesar de abastecerse de madera proveniente de bosques naturales.

Si atendemos ahora a Seele y Gatti (2015, citado en Salas, 2018), podría decirse que estamos ante un caso de "greenwashing genuino" como respuesta a los escándalos desvelados por una variedad de ONG que acusana AP\&P mediante pruebas evidentes, perjudicando así la imagen de la misma.

Porúltimo, según la clasificación del concepto de greenwashing de Greenpeace (Alejos, 2013, p.8) que incluye cuatro tipos, el caso de $A P \& P$ se enmarcaría como: Negocio sucio (dirtybusiness), pues promueveactividades insostenibles y contaminantes en gran parte de Indonesia, siendo poco transparente.

En resumen, la alianza de Greenpeace, WWF, Geoinnova y otras ONG locales, utilizando técnicas del marketing ecologista lograron visibilizar el impacto ambiental de AP\&P y la responsabilidad de Mattel y otras empresas jugueteras. Desmontando la incoherencia entre su discurso verde y sus prácticas lesivas al medio ambiente, esto es, la práctica de greenwashing, las ONG ambientalistas forzaron a las empresas a realizar cambios reales, impulsándolas a adoptar iniciativas del marketing ecológico. Aunque con el posicionamiento actual de AP\&P se hayan dado una serie de acciones que le acercan al marketing ecológco, aún le quedan muchos asuntos pendientes que solucionar y continúa con una estrategia incoherente en la que se intercalan avances reales con campañas de greenwashing. Así, la consulta del apartado Sustainability de la web de AP\&P (s.f. a) muestra claramente una imagen verde de la empresa en la que se recopilan múltiples compromisos y avances sin mencionar en ningún momento los escándalos en los que se ha visto envuelta y sin dar relevancia tampoco a los múltiples retos pendientes.

\section{CONCLUSIONES}

El caso de Mattel y Asia Pulp \& Paper, así como las denuncias de ONG como Greenpeace, WWF o Geoinnova, ilustra la interrelación entre los conceptos expuestos de marketing ecológico, marketing ecologista y greenwashing. Por una parte, la acción de las ONG ambientalistas ejemplifica el uso del marketing ecologista, un tipo de marketing social. Las ONG han empleado para ello herramientas como la investigación, acciones de street marketing, la publicidad (o concretamente, contrapublicidad), relaciones con la prensa, campañas de presión y lobby, etc. A menudo, estas campañas se han dirigido estratégicamente a stakeholders relevantes para AP\&P, en especial Mattel como gran cliente de AP\&P. Aprovechando la gran notoriedad de Mattel y de su marca icónica Barbie, ONG como Greenpeace han hecho uso de campañas de contrapublicidad muy efectivas, ya que precisamente esa notoriedad de las marcas las hace vulnerables a las denuncias, que obtienen también gran repercusión y por tanto causan un importante perjuicio a la imagen corporativa (Klein, 2001). Esta estrategia incide en el punto más débil de AP\&P como proveedora de las grandes marcas, logrando incluso que grandes clientes de AP\&P más expuestos a la opinión pública española rompan sus relaciones comerciales con la papelera, lo que supone para $A P \& P$ un gran coste no solo de imagen, sino también económico (Mustiko \& Sutikno, 2015; Majláth, 2016;).

Por su parte, AP\&P o Mattel, tanto antes como después de la campaña, han usado estrategias ligadas al greenwashing, con distintas modalidades como la prueba faltante, el concepto ambiguo y sobre todo la autoglorificación. Esta estrategia puede afectar a la imagen e incluso a la intención de compra de los consumidores potenciales (Akturan, 2018; Schmuck, Matthes \& Naderer, 2018). Pero, además, la presión ejercida por las ONG ha obligado a las empresas a llevar a cabo cambios reales aunque insuficientes en relación a su política empresarial, impulsándolas a adoptar el respeto al medio ambiente como eje transversal de las compañías e incorporarlo a una incipiente estrategia de marketing ecológico.

Con respecto a sus valores actuales, AP\&P afirma que apuesta por ofrecer innovación de manera sostenible y responsable con la intención de crear un mundo mejor y declaran que "Ios 
principios del liderazgo residen en la construcción de un negocio sostenible" (AP\&P, s.f. b). A pesar de los esfuerzos comunicativos, AP\&P tiene el reto de restaurar su imagen corporativa. Las primeras reacciones de la compañía, acusando a Greenpeace de denuncias infundadas, de "jugar con los sentimientos de los niños" (sic) o de atacar a una nación en vías de desarrollo (Comunicarse, 2011) han mostrado que esta es una estrategia errónea y condenada al fracaso. Además de no lograr rebatir las acusaciones de la ONG y evitar el daño de su imagen corporativa, $A P \& P$ ha tenido que asumir el importante coste de perder grandes clientes (Greenpeace, 2011)y finalmente no ha tenido más remedio que asumir también el coste del cambio hacia una política empresarial más enfocada al marketing ecológico.

AP\&P ha tomado una actitud reactiva que cuestiona la tradición, la teoríay las buenas prácticas de las relaciones públicasy la verdadera responsabilidad social corporativa. Consideramos que este caso ejem plifica que marketing ecológco resulta una estrategia más adecuada a largo plazo y que muchas em presas deberían aprender del caso estudiado. La lección de AP\&P es que no sirve de mucho tratar de frenar lo inevitable. En un mundo cada vez más amenazado por el cambio climático, crece también la conciencia ambiental de buena parte de la población, sus demandas como consumidores, el valor añadido de los productos ecológicos y, también, el avance de la legislación proteccionista. Una actitud proactiva por parte de las empresas para adoptar estrategias de marketing ecológico parece una apuesta de futuro que, a pesar de sus costes iniciales, resultaría más rentable entérminoseconómicos y de imagen y por supuesto más responsable social y ambientalmente. Siendo así, el futuro de las empresas también está ligado al futuro del planeta aunque, en cualquier caso, la sociedad puede permitirse prescindir de aquellas empresas que no estén dispuestas a colaborar, no del planeta.

\section{REFERENCIAS BIBLIOGRÁFICAS}

- Akturan, U. (2018). How does greenwashing affect green branding equity and purchase intention? An empirical research. Marketing Intelligence \& Planning, 36(7), 809-824. DOI: https://doi.org/10.1108/MIP-12-2017-0339.

- Alejos, C.L. (2013). Greenwashing. Ser verde o parecerlo. Cátedra CaixaBank de Responsabilidad Social Corporativa, no 23. https://media.iese.edu /research/pdfs/ST0328.pdf.

- Andreasen, A.R. (1994). Social marketing: Its definition and domain. Journal of public policy \& marketing, 13(1), 108-114. DOI: https://doi.org/10.1177/074391569401300109.

- Andreasen, A.R. (1996). Profits for nonprofits: Find a corporate partner. Harvard Business Review, 74(6), 47-59. https://pubmed.ncbi.nlm.nih.gov/10162358/.

- AP\&P (s.f. a). Sustainability. https://asiapulppaper.com/sustainability.

- AP\&P (s.f. b). About us. https://asiapulppaper.com/about-us.

- Asociación Española de Fabricantes de Juguetes. (s.f.). MATTEL ESPAÑA, S.A. https://www.aefj.es/asociados/mattel- espana-sa.

- Bartels, R. (1974): The Identity Crisis in Marketing. Journal of Marketing, 38(4), 73-76. DOI: https://doi.org/10.1177/002224297403800413.

- Calomarde, J. V. (1995). Influencia de los factores ambientales en la decisión de compra de bienes de consumo. ESIC Market, (89), 125-154.

- Calomarde, J. V. (2000). Marketing ecológico. Pirámide.

- Castells, M. (2009). Comunicación y poder. Alianza.

- Chamorro Mera, A. (2001). El Marketing Ecológico. 5campus.org.

http://www.5campus.org/leccion/ecomark eting.

- Comunicarse. (2011, 16 de junio). Mattel prepara una nueva política de envases luego de la denuncia de Greenpeace.

https://www.comunicarseweb.com/biblioteca/ma ttel-prepara-una-nueva-politica-de-envases-luegode-la-denuncia-de-greenpeace.

- Coddington, W. (1993). Environmental marketing: positive strategies for reaching the green consumer. McGraw- Hill.

- de Lestrade, T. (2012). Greenpeace, the story [película]. Film Ideas.

- Europa Press (2011, 9 de junio). La proveedora de los envoltorios de 'Barbie'se defiende de las acusaciones de Greenpeace sobre destrucción de selvas.

https://m.europapress.es/epsocial/rsc/noticiarsc-proveedora-envoltorios-barbie-defiendeacusaciones-greenpeace-destruccion-selvas20110609143850.html.

- Flyvbjerg, B. (2006). Five Misunderstandings About Case-Study Research. Qualitative Inquiry, 
12(2), 219-245. DOI:

https://doi.org/10.1177/1077800405284363.

- García Marzá, D. (1999) La responsabilidad ecológica de la empresa: El punto de vista de la ética empresarial. En Gonzales, E. (ed.). Ética y ecología: La gestión empresarial del medio ambiente (pp. 17-29). Universitat Jaume I.

- Geoinnova (s.f.) Forest Heroes y su lucha contra la deforestación en Indonesia.

https://geoinnova.org/blog-territorio/forestheroes-y-su-lucha-contra-la-deforestacion-enindonesia/.

- González, N. (2011, 21 de junio) En vivo/ Greenpeace pide a Disney y Hasbro que no colaboren con la deforestación [mensaje en un blog]. http://archivo-

es.greenpeace.org/espana/es/Blog/en-vivogreenpeace-pide-a-disney-y-hasbroque/blog/35394/.

- Greenpeace (2008). The Greenpeace Book of Greenwash.

https://research.greenpeaceus a.org/?a=view\&d= 4588.

- Greenpeace (2011a, 7 de junio). Ken rompe con Barbie (Greenpeace contra la deforestación en Indonesia).

https://www.youtube.com/watch?v=kO_zpLUOE $\mathrm{Mg}$.

- Greenpeace (2011b, 8 de junio). Greenpeace vincula al fabricante de la muñeca Barbie con la deforestación en Indonesia. http://archivoes.greenpeace.org/espana/es/n ews/2011/June/Gr eenpeace-vincula-al-fabricante-d e-la-munecaBarbie-con-la-deforestacion-en-Indonesia/.

- Greenpeace (2011c, 21 de junio). Acción contra la deforestación en Hasbro, Mattel y Disney. [Archivo de vídeo]. https://cutt.ly/hyCMTOD.

- Greenpeace. (2011d, 5 de octubre). Barbie pone fin a su relación con la deforestación de las selvas de Indonesia. http://archivo-

es.greenpeace.org/espana/es/n ews/2011/October /Barbie-pone-fin-a-su-relacion-con-ladeforestacion-de-las-selvas-de-Indonesia/.

- Greenpeace (s.f.). Salvemos los bosques de Indonesia. https://es.greenpeace.org/es /quepuedes-hacer-tu/peticiones/salvemos-bosquesindonesia/.

- Hart, C. (2008). Doing a literature review: Releasing the social science research imagination. Sage.

- Klein, N. (2001). No logo. El poder de las marcas. Paidós.

- Koalisi Anti Mafia Hutan (2019). AP\&P acknowledges links to controversial suppliers, but fails to release an auditor's report. http://d2ouvy59p0dg6k.cloudfront.net/download s/asia_pulp__paper_acknowledges_links.pdf.

- Kotler, P. y Roberto, E.L. (1991). Marketing social. Estrategias para cambiar la conducta pública. Díaz de Santos.

- Kotler, P. \& Zaltman, G. (1971). Social Marketing: An Approach to Planned Social Change. Journal of Marketing, 35(3), 3-12. DOI: https://doi.org/10.2307/1249783.

- Kotler, P. \& Levy, S. (1969). A New Form of Marketing Myopia: Rejoinder to Professor Luck. Journal of Marketing, 33(3), 55-57. DOI: https://doi.org/10.2307/1248483.

- Leal, A. (2000). Gestión del marketing social. McGraw-Hill.

- Leal, A. (2004). El Marketing Social en España: Situación actual y estrategias para su desarrollo. Revista Internacional de Marketing Público y No Lucrativo, 1(1), 32-52.

https://dialnet.unirioja.es/servlet/articulo?codigo $=1418770$.

- Luck, D. J. (1974). Social Marketing: Confusion Compounded. Journal of Marketing, 38(4), 70-72. DOI: https://doi.org/10.1177/002224297403800412.

- MacDonald, K. \& Tipton, C. (1993). Using documents. Sage.

- Majláth, M. (2016). How does greenwashing effect the firm, the industry and the society - The case of the VW emission scandal. 16 Symposium for Young Researchers, Budapest, Hungría. https://kgk.uniobuda.hu/sites/default/files /12_Maj1\%C3\%A1th.p df.

- Maitar, B. (5 de febrero de 2013). Asia Pulp \& Paper se compromete a poner fin a la deforestación [mensaje en un blog]. http://archivoes.greenpeace.org/espan a/es/Blog/victoria-asiapulp-paper-se-compromete-a-pone/blog/43861/.

- Martínez Carazo, P. C. (2006). El método de estudio de caso. Estrategia metodológica de la investigación científica. Pensamiento y Gestión, (20), 165-193.

https://www.redalyc.org/articulo.oa? $\mathrm{id}=6460200$ 5.

- Mattel (2011, 5 de octubre) Mattel Announces Sustainable Sourcing Principles. https://mattel.gcsweb.com/static-files/972e82da-a2 3f-4f59-b42 68084d3c19d2f.

- Mattel (2019). Mattel Announces Goal to Achieve 100\% Recycled, Recyclable or Bio-based Plastic Materials in All Products and Packaging By 2030. https://corporate.mattel.com/news/mattelannounces-goal-to-achieve-100-recycled- 
recyclable-or-bio-based-plastic-materials-in-allproducts-and-packaging-by-2030.

- Mattel. (s.f.). Diversity. https://corporate.mattel.com/aboutus/diversity.aspx.

- McCarthy, J. E. \& Perreault, W. D. (1984). Basic Marketing: a managerial AP\&Proach. R.D. Irwin.

- Montero Simo, M. J. (2003). El marketing en las ONGD. La gestión del cambio social. Ediciones Universidad de Navarra.

- Muñoz Serra, V.A. (2013). Marketing ecológico. Universidad de Concepción. http://repositorio.udec.cl/jspui/bitstream/11594/ 1766/1/marketing_\%20ecologico.Image.Marked.p df.

- Mustiko, H.y Sutikno, B. (2015). The extended consequence of greenwashing: Perceived consumer skepticism. International Journal of Business and Information, 10(4), 433-468. DOI: 10.6702/IJBI.2015.10.4.2.

- Peattie, K. (1995). Environmental Marketing Management. Pitman.

- Pechmann, C. (2002). Psicología y marketing. Editorial Iberoamericana.

- Pérez Romero, L. A. (2004). Marketing social. Teoría y práctica. Pearson Educación.

- Pistilli, G. (2015). Il green marketing: la comunicazione della sostenibilità. [tesis de maestría, Libera Università Internazionale degli Studi Sociali Guido Carli]. Luiss Thesis. http://tesi.eprints.luiss.it/16677/.

- Salas Canales, H.J. (2018). El greenwashing y su repercusión en la ética empresarial. Neumann Business Review, 4(1), 28-43. DOI: https://doi.org/10.22451/300 2.nbr2018.vol4.1.10 018.

- Santesmases Mestre, M. (2012). Marketing: Conceptos y estrategias. Pirámide.

- Schmuck, D., Matthes, J. \& Naderer, B. (2018). Misleading Consumers with Green Advertising? An Affect-Reason-Involvement Account of Greenwashing Effects in Environmental Advertising. Journal of Advertising , 47(2), 127-145. DOI: https://doi.org/10.1080/00913367.2018.1452652

- Seele, P. \& Gatti, L. (2015). Greenwashing Revisited: In Search of a Typology and AccusationBased Definition Incorporating Legitimacy Strategies. Business Strategy and the Environment, 26(2), 239-252. DOI: https://doi.org/10.1002/bse.1912.

- Siano, A., Vollero, A., Conte, F. \& Amabile, S. (2017). "More than words": Expanding the taxonomy of greenwashing after the Volkswagen scandal.
Journal ofBusiness Research, 71, 27-37. DOI: https://doi.org/10.1016/j.jbusres.2 016.11.002.

- Soto, M.A. (2011a, 14 de junio). Mattel no reacciona [mensaje en un blog]. http://archivoes.greenpeace.org/espana/es/Blog/mattel-noreacciona/blog/35273/.

- Soto, M.A. (2011b, 5 de octubre). Barbie y Mattel dicen no a la deforestación [mensaje en un blog]. http://archivoes.greenpeace.org/es pana/es/Blog/ barbie-y-mattel-dicen-no-aladeforestacin/blog/37186/.

- Wicke, L. (1992). Chancen und Probleme der Betriebe durch umweltfreundliche Produkte. En Hopfenbeck, W. (ed.) Dirección y Marketing ecológicos. Deusto.

- WWF (2018). Joint NGO Statement on 5th Anniversary of Asia Pulp \& Paper's Forest Conservation Policy.

http://awsassets.panda.org/downloads/Joint_NGO _statement_on_AP\&P_FCP_5th_anniversary_(05F eb 18)_FINAL_logos.pdf.

- WWF (s.f. a). Una especie en creciente declive por la deforestación.

https://www.wwf.es/nuestro_trabajo/especies_y_ habitats/orangutan/.

- WWF (s.f. b). History of deforestation by AP\&P/April.

https://wwf.panda.org/our_work/our_focus/fores ts_practice/forest_sector_transformation_updated/ AP\&P_april_updated/deforestation_upd ated/.

- WWF et. al. (s.f.) Eyes on the Forest. https://www.eyesontheforest.or.id/about.

- Yin, R. (2003). Case Study Research: Design and Methods. Sage. 